Optimizing the Scientific Study of Suicide with Open and Transparent Research Practices

Thomas P. Carpenter, Ph.D.

Keyne C. Law, Ph.D.

Seattle Pacific University

Keywords: Suicide, Reproducibility, Open Science

Correspondence concerning this article should be addressed to Keyne C. Law, Department of Clinical Psychology, Seattle Pacific University, 3307 3rd Ave West, Seattle WA 98119-1997.

Email: 1awk3@spu.edu 


\begin{abstract}
Suicide research is vitally important, yet—like psychology research more broadly—faces methodological challenges. In recent years, researchers have raised concerns about standard practices in psychological research, concerns that apply to suicide research and raise questions about its robustness and validity. In the present paper, we review these concerns and the corresponding solutions put forth by the 'open science' community. These include using open science platforms, pre-registering studies, ensuring reproducible analyses, using high-powered studies, ensuring open access to research materials and products, and conducting replication studies. We build upon existing guides, address specific obstacles faced by suicide researchers, and offer a clear set of recommended practices for suicide researchers. In particular, we consider challenges that suicide researchers may face in seeking to adopt 'open science' practices (e.g., prioritizing large samples) and suggest possible strategies that the field may use in order to ensure robust and transparent research, despite these challenges.
\end{abstract}




\section{Optimizing the Scientific Study of Suicide with Open and Transparent Research Practices}

The suicide research community is united under the common goal of preventing suicide deaths. There are many ways to approach this goal, with some focusing on building theories for better understanding suicide, some developing and disseminating evidence-based interventions, and others improving assessment and prediction of suicide risk and behavior. To reach this goal, policy makers, clinicians, and researchers rely heavily on suicide research, making the validity and reliability of the suicide literature essential for researchers and the public alike. A metaanalysis evaluating the last 50 years of suicide research, however, found that the ability to predict suicide has not improved, despite the immense resources that have gone into this research (Franklin et al., 2017). At the same time, research into the replicability of psychological research (Open Science Collaboration, 2015) raises serious questions about the standard practices surrounding how research is designed, executed, analyzed, and published. Critically, these questions do not assume bad faith on behalf of researchers; instead, they suggest that concerns long voiced by methodologists (e.g., low power, publication bias, overreliance on $p<.05$ ) have real impacts on the trustworthiness of the literature. In response, the 'open science' community has offered a set of practices and considerations designed to address these issues, which have been adopted by a growing number of journals (e.g. Science, Nature, and Psychological Science) and funding agencies. In this paper, we review these issues as they pertain to suicide research, explore open-science solutions, and offer resources for suicide researchers to improve the robustness and replicability of their empirical work. 


\section{The Replication "Crisis"-Or, Reasons for Concern}

There is no need to change practices if current results are satisfactory. Psychological research generally uses samples to makes claims about populations, often via $p$-values (Neyman \& Pearson, 1933); these $p$-values are the basis of much of the suicide literature. Unfortunately, $p$ values are often misunderstood (e.g., Badenes-Ribera et al., 2016). They do not indicate the probability that a result is a false positive; they indicate the proportion of the time that a result as large (or larger) than that observed would occur if the null hypothesis was true (Greenland et al., 2016). Thus, $p=.02$ indicates disagreement between the data and the null hypothesis; however, it does not mean that there is only a $2 \%$ probability that the null hypothesis is true (Greenland et al., 2016). That probability could be much higher.

For example, Simmons, Nelson, and Simonsohn (2011) showed that researchers regularly inflate their false positive rates, which can reach as high as $60 \%$ using common research practices, such as swapping outcomes to focus on significant results, adding participants to see if trending findings 'pan out,' and adding moderators after looking at the data $-\mathrm{a}$ family of practices they termed " $p$-hacking." Since these decisions are made after inspecting results from a given sample, they are especially likely to capitalize on chance. When combined with factors such as publication bias (where null results are not published), low statistical power (inability to detect true relationships), and a desire for novelty (the study tests a hypothesis about which truth is questionable prior to seeing the data), it is possible to end up with a literature filled with false positives (Ioannidis, 2005). This is particularly unhelpful when our goal is to improve suicide risk prediction and suicide prevention practices.

A simple example illustrates this problem. Consider a researcher who tests 100 risk factors for suicide - some of which are "true effects" and some of which are not. Assuming the 
researcher is right $30 \%$ of the time, there should be 30 true effects and 70 non-effects. How good are current research practices at identifying these true effects? Unfortunately, low power keeps many from being discovered. Median power for medium-size effects in psychology (not suicidespecific) is estimated at $44 \%$ and is worse for small effects (12\%; Szucs \& Ioannidis, 2017). Assuming a medium effect size, a minority of true effects will be detected $(44 \%=13.2$ true positives). Meanwhile, $5 \%$ of the 70 null effects (3.5 tests) are expected to be false positives. False positives thus make up $21 \%$ of all significant results (3.5 false positives, 13.2 true positives $)^{1}$ — owing to the fact that "true positives" are infrequent. Worse, if null results are not published and replication studies are not performed, there is no record to refute these false positives. Further, anything that decreases the true positive rate (low power, unlikely hypotheses, many exploratory analyses) or increases false positives ( $p$-hacking) will worsen the problem.

There are no published estimates of replicability for suicide research. However, empirical estimates in the broader psychology literature suggest that false positives are common, with replicability estimated at only $36 \%-54 \%$ on the basis of large, multi-lab replication studies (e.g., Dang et al., 2019; Ebersole et al., 2016; Forscher et al., 2019; Hagger et al., 2016; O’Donnell et al., 2018; Open Science Collaboration, 2015; Wagenmakers et al., 2016). Unless we believe that suicide research has better practices than psychology at large (e.g., higher power, better theory, less $p$-hacking, etc.), then there is reason for concern about the state of suicide research as well. Estimating replicability (e.g., replication studies, large-scale multi-lab studies) for suicide research is an important first step in assessing our research practices. However, assuming that they are similar to the field at large, then it is necessary for us to collectively improve our research practices to ensure the reproducibility of our work.

\footnotetext{
${ }^{1}$ This is known as the false discovery rate (FDR; Benjamini \& Hochberg, 1995)
} 


\section{Reasons for the Problem}

Replication alone is not sufficient to remedy the replication crisis, particularly in the context of observational epidemiologic studies and clinical trials that are often used in suicide and suicide prevention research (see Coyne, 2016). If we seek to have a robust and truthful literature in suicide research, it is necessary to understand sources of bias and avenues to address them, collectively referred to as "open science" (Nosek \& Bar-Anan, 2012; Nosek, Spies, \& Motyl, 2012; Uhlmann et al., 2019). Several manuscripts have been written to provide guidance to researchers on how to adopt these research practices (Klein et al., 2018) and apply them to clinical psychology (Tackett et al., 2017). They do not, however, address the issues specific to suicide research. We next discuss the issues underlying the replication crisis and practices that may address these issues (Crüwell et al., 2018; Back, Hendriks, \& Schönbrodt, 2017), as well as obstacles unique to suicide research.

Issue 1: File Drawer / Publication Bias. Recent meta-analytic work suggests there exists substantial publication bias in suicide research (Franklin et al., 2017). Publication bias is the phenomena that findings do not get published, biasing the literature in favor of findings that do get published. Specifically, Franklin et al. (2017) reviewed 50 years of studies predicting suicidal ideation, suicide attempts, and suicide deaths_-finding "high" to "moderate" degrees of publication bias toward omitting non-significant results using several metrics of publication bias. After adjusting for publication bias, the meta-analysis uncovered that many risk factors reported in extant suicide literature are weak or questionable, and the authors found the data provided little insight on how to advance research and prevention practices. Indeed, they found that — after adjusting for publication bias — effect sizes for predictors of ideation decreased by $40 \%$, and 
effect sizes for predictors of suicide behavior decreased by $50 \%$. This suggests that publication bias may be a substantial problem for the validity of the suicide-prediction literature.

In general, publication bias distorts the validity of a literature. Some kinds of publication bias may be good - for example, filtering out methodologically flawed papers or analyses. Publication bias, however, tends to favor certain kinds of findings, such as statistically significant and novel results (e.g., Easterbrook, Gopalan, Berlin, \& Matthews, 1991). By censoring other results, publication bias makes the literature non-representative. Consider a researcher reporting the results of a coin toss_-if all "heads" results are unpublished, then the publication record for the coin is $100 \%$ "tails." Similarly, if a weak effect size is more difficult to publish than a large effect size, then effect sizes in the published literature will be biased upwards - exactly the pattern seen in replication research (Open Science Collaboration, 2015) and the pattern observed by Franklin et al. (2017) in suicide research. Although there are metaanalytic techniques to address these issues (e.g., trim and fill), they all fail under various conditions (Carter, Schönbrodt, Gervais, \& Hilgard, 2019). The best solution, then, is to not censor results because of the results themselves (Nosek \& Bar-Anan, 2012).

Some may argue that null results are ambiguous. After all, a null result may indicate a true null hypothesis or lack of power to detect some small effect (Greenland et al., 2016; Kirk, 2008). Thus, interpreting $p>.05$ is difficult. Modern data analysis tools can address this criticism. With large samples, effect sizes can be precisely estimated. For example, if the effect of a treatment on depression is $d=.01,95 \%$ CI $[-.09, .11]$, then this "null" result has precisely estimated a "zero or trivially small" effect (for more examples, see Dang et al., 2019; Hagger et al., 2016). Newer statistical tools such as equivalence testing use this same information to provide a test of support for the null hypothesis (Lakens, 2017); Bayesian analysis similarly 
provides measures of relative support for the null and alternative hypotheses as well (Masson, 2011). Thus, being "null" does not automatically make a result uninformative. A null result that precisely estimates an effect as trivial or close to zero can indeed have great theoretical and practical importance. Achieving such a conclusion, however, typically requires large samples and the use of as confidence intervals, equivalence testing, or Bayesian analyses. As discussed below, the need for large sample is particularly problematic in suicide research, where base rates are low and participants may be difficult to study (Belsher et al., 2019). Nevertheless, this does not negate its importance.

The value of a null result may depend in part on the question asked. One premise of science is falsifiability - the ability to reject ideas that do not accurately describe the world (LeBel et al., 2017; Popper, 1963). Given that false positives do occur, it is vital that researchers can correct the literature. If a theory makes a prediction that is not well supported, then it is important that researchers publish these findings. If, for example, a researcher testing the interpersonal theory of suicide (ITS; Joiner, 2005) is unable to publish findings predicted by the theory because their effect sizes are non-significant or small, then science is rendered incapable of refuting the theory. We contend, therefore, that if a study extends logically from theories or ideas that are believed to be true $a$ priori, and a study finds evidence against those ideas, then the study has evidentiary value. Selectively publishing only 'positive' results only muddies the waters. Indeed; making null result available may prevent redundant work in multiple laboratories; given that suicide research is expensive and resources are scarce, it is unwise to use participants' and researchers' time and emotional labor to test hypotheses if evidence exists against that hypothesis. In contrast, a study that tests a purely novel idea — and returns no evidence for it - may not be as important for the literature. 
Open science solutions. Two solutions to publication bias are "results blind" peer review and registered reports (Grand et al., 2018). In results-blind peer review, papers are first reviewed with their results omitted, forcing reviewers to assess the quality of the question and rigor of the methods. An extension of this approach is the registered report format (Chambers et al., 2014; Grand et al., 2018), in which the paper undergoes peer review prior to data collection—with acceptance offered based on the question and methodology. As articulated by Merton (1942), one fundamental principle of science is dispassion. If scientists wish to provide accurate and replicable research, we must constrain our inner biases, hopes, and desires and provide an accurate and objective view of our data. These methods provide one way of restraining those biases; however, as discussed below, the methodology community has suggested others as well.

Another solution is for researchers to provide open access to their research products free of cost, including peer-reviewed and non-peer reviewed journal articles, conference papers, theses and dissertations, book chapters, datasets, and more. Researchers can publicly post their manuscript before formal peer-review, known as a pre-print, in repositories such as OSF Preprints and PsyArXiv (endorsed by APA). In addition, the Open Science Framework (www.osf.io) provides tools for the archival of datasets and analysis code, research protocols, and more. One benefit of preprinting is that authors can receive feedback before submission to a peer-reviewed journal. Pre-prints also receive a DOI and timestamp allowing them claim their ideas should others attempt to publish them. The rules on the self-archival varies across journals, although most allow some version of self-archival. ${ }^{2}$

Issue 2: p-Hacking and HARKing. $p$-hacking (Simmons et al., 2011) is a process whereby many analyses are run on data with the aim of finding an analysis that produces $p<.05$.

\footnotetext{
${ }^{2}$ Policies can be browsed here prior to submitting http://www.sherpa.ac.uk/romeo/index.php
} 
Once this is found, the researcher may then "Hypothesize After Results are Known," or HARK (Kerr, 1998). The end result is a paper that appears to have made a precise a priori prediction. In reality, the result is one of many attempts at a significant result. As discussed below, this invalidates the use of $p$-values (Greenland et al., 2016).

The degree to which $p$-hacking and HARKing occur in suicide research is unknown. However, they are broadly prevalent in clinical science and science more broadly (Head et al., 2015). Further, analysis of clinical trials before and after 2000 (when preregistration became the norm in the United States) suggests that $p$-hacking was widespread (Kaplan \& Irvin, 2015). Specifically, when authors were required to adhere to a pre-registered analysis plan, support for interventions dropped from $57 \%$ to $8 \%$ in the literature. Finally, it has been part of the training model in psychology for years. For example, Bem (1987, p. 171-172) advocated for this strategy in a manual for young psychologists:

There are two possible articles you can write: (a) the article you planned to write when you designed your study or (b) the article that makes the most sense now that you have seen the results. They are rarely the same, and the correct answer is (b).... Examine [the data] from every angle. Analyze the sexes separately. Make up new composite indexes. If a datum suggests a new hypothesis, try to find additional evidence for it elsewhere in the data. If you see dim traces of interesting patterns, try to reorganize the data to bring them into bolder relief. If there are participants you don't like, or trials, observers, or interviewers who gave you anomalous results, drop them (temporarily). Go on a fishing expedition for something - anything - interesting.

Although it is unknown to what degree these practices occur in suicide research, there are several reasons why variants of these practices may be tempting to suicide researchers. Suicide 
studies - particularly grant-funded projects — can be expensive and take considerable time to design and execute. As such, researchers are likely to continue thinking after a study is conceived and may have considerable time to do so (sometimes years). Consequently, the final use of the data may deviate from the initial plan when a study was designed. Analysis decisions may also vary: researchers also have to consider the aspect of suicide they would like to examine (i.e. suicidal ideation vs. suicide attempts) and even subtle nuances in definition such as whether or not they should count aborted or interrupted attempts as suicide attempts. Additionally, researchers often must consider whether it would be appropriate to control for psychopathology (e.g. depression) when testing models for suicide. Further, if researchers believe that null results are not publishable, then it may be tempting to test many different questions (or permutations of the same question) to ensure the data and funding to not go to waste. Although having multiple uses for a single dataset is not necessarily problematic, it may be tempting to inadvertently run many analyses and —inadvertently or intentionally—selectively publish those reach $p<.05$.

As noted earlier, Simmons et al. (2011) have shown that such strategies cause false positive rates to balloon. At issue is the fact that the researcher does not have population data; thus, when a given finding is significant, it is unclear whether the result is due to chance. False positives should occur roughly $5 \%$ of the time a researcher is wrong; if researchers ask many questions of a dataset, false positives will eventually appear. Thus, a low $p$-value is not support for a hypothesis if it took many analyses to find it (Greenland et al., 2016). Further, attempts to follow-up false positives in the same dataset will be likely to confirm the false positive, as the issue is in the non-representativeness of the data with respect to the question asked. Simmons et al. (2011) articulated many such $p$-hacking strategies, including exploring moderators (e.g., gender), adding additional participants after inspecting the data, dropping experimental 
conditions from a study, and adding multiple dependent variables. As noted earlier, false positive rates can reach $60 \%$.

Although it may be tempting to ascribe such strategies to bad actors, this need not be the case. As argued by Simmons et al. (2011), researchers have multiple "degrees of freedom" in their analysis approach; when two defensible approaches yield different conclusions, it may be tempting to selectively report the approach that yields the desired conclusion. In a dramatic display of this, Silberzahn et al. (2018) had 61 data analysis teams assess the same hypothesis across the same (complex) dataset and found that $69 \%$ of teams found significant evidence for the effect, whereas $31 \%$ did not. Further, reviewer assessments of the credibility of approaches could not account for these discrepancies. At least in cases of complex data, it may simply be the case that there are many defensible ways to operationalize and test a prediction-yielding potentially different conclusions.

HARKing, particularly undisclosed HARKing, further complicates the issue. When a study is HARKed, the researcher changes their prediction to match the results of the study, often without disclosure. This can happen both after exploratory analyses (where false positives are more likely) or not. If this was not disclosed, then the paper provides no indication that the result was not truly a confirmatory test of a hypothesis; it also does not provide the information necessary to contextualize the $p$-value (i.e., fails to report that the significant result was one of several predictions tested). Although it is possible that a HARKed study was optimally designed to test whatever post-hoc hypothesis was reported, this will not always be the case. We discuss potential solutions to these issues below.

Open science solutions. We agree that exploratory or unplanned analyses are valuable and necessary in science. In such cases, however, we argue that there is simply a missing step. 
There is no way to confirm post-hoc speculations generated by a dataset on the same dataset. Null hypothesis testing is a confirmatory technique; if a study suggested an idea, there is nothing wrong with describing it as such and following up with a second strictly confirmatory sample. For example, Werner et al., (2019) used such a format to identify the role of 'grit' in academic achievement using a novel statistical technique as part of a larger study; because that analysis was not initially planned, it was then directly replicated in a second sample — and described as such.

How can researchers assure themselves and others that a finding was not $p$-hacked? One option is preregistration of analysis plans and hypotheses (van 't Veer \& Giner-Sorolla, 2016) which increases transparency and limits flexibility during the data analytic process $(\mathrm{Pu}, \mathrm{Zhu}$, Kay, \& Conrad, 2019). Given that the NIH established the expectation that all clinical trials funded by the NIH are registered on ClinicalTrials.gov in 2017 (National Institutes of Health, 2016), this practice has been adopted by clinical trial researchers with approximately 512 trials for suicide-focused treatments registered. However, even studies that are not clinical trials can be pre-registered. To pre-register their study, researchers produce a document specifying a study's hypotheses and data analytic plans to a repository that time-stamps the predictions (e.g., www.aspredicted.org) prior to collecting data. This ensures that researchers clearly differentiate pre-registered, confirmatory findings from findings that arose during data-dependent exploratory analyses.

A pre-registration requires that researchers specify their analysis plans up front, requiring that researchers think through analyses at a level of detail they might typically skip until data are collected (van 't Veer \& Giner-Sorolla, 2016). Especially in complex datasets, there may be multiple ways to operationalize a conceptual hypothesis. Because of this, one way to 
troubleshoot problems before data analysis is to simulate some number of rows of artificial data to ensure that the pre-specified analyses make conceptual sense for the data that will be collected. Although generating a this can be done by hand, a number of tools exist that aid in this process (e.g., https://debruine.github.io/faux/). Given the increasing use of Experience Sampling Methodology (ESM) and Ecological Momentary Assessment (EMA) in studying idiosyncratic suicide risk factors, Kirtley and colleagues (2019) have developed a pre-registration template specifically for ambulatory assessments. We encourage suicide researchers to share preregistration templates for various techniques as they develop them.

Like $p$-values, pre-registration is a confirmatory tool; it assures the reader that the study did (only) what it set out to do. This many not always be desired. For example, a researcher examining a new predictor of suicidal ideation may wish for the flexibility to examine it in multiple ways, with and without adjusting for various covariates, with and without various mediators or moderators, etc. Such analyses play an important role in science and allow the researcher to explore the data; they may also not be the best candidates for pre-registration. However, researchers should be aware that such analyses also prohibit one from treating significant $(p<.05)$ results as strong evidence for the confirmation of a hypothesis (Greenland et al., 2016). Following Werner et al. (2019), one strategy for this is the 'exploratory-confirmatory' paper (or set of papers). Once an interesting result is discovered, an independent confirmation in a novel sample can be pre-registered and run to ensure that the result is robust and replicable.

A common barrier to preregistration is the belief that preregistration makes research inflexible and does not allow for changes. The preregistration document is not meant to be a binding contract but rather a guide that transparently reports the decisions made by researchers. Once preregistrations are submitted, researchers are not able to modify the submitted document. 
However, in the interest of good science, the researcher may still make changes to their procedure. Especially when testing a novel question, a researcher reach the analysis stage only to discover that a planned analysis was poorly specified or illogical, or perhaps another statistical procedure may better suit the data. In this case, the researcher should prioritize doing good science, document these deviations, and include them with the manuscript (in the text or as a supplement). In addition, there is no reason why a pre-registered study cannot include exploratory analyses as well, so long as they are labelled as such. When going "off the grid" of one's planned analyses, we suggest that researchers transparently report all relevant analyses conducted to avoid selective reporting. Importantly, this provides a record of what was done.

We see three major challenges for implementing preregistration in suicide research. First, suicide data can be expensive and difficult to obtain, and the field thus often relies on archival data. Specifically, researchers often include many measures when collecting data so that hypotheses generated later can be tested. One way to prevent accidentally $p$-hacking results in such cases is to specify analysis plans before testing them in the data, even if the data are preexisting. Although preregistration prior to data collection is ideal, Weston et a., (2018) offer numerous resources for secondary data analysis (see also their repository https://osf.io/x4gzt/). For further discussions of preregistration for existing and archival data, see Mertens and Krypotos (2019).

A second challenge for preregistration in suicide is that it may be difficult to 'confirm' an exploratory result in a novel sample when novel data are difficult to obtain. However, this does not negate the importance of confirming important findings - although this may be left to replication studies. For example, Nock et al., (2010) found initial evidence for the predictiveness of implicit cognition in suicide attempts. A decade later, Tello et al. (2020) published a pre- 
registered direct replication (which confirmed the finding, but with a smaller effect size). These replications are important; given the issues discussed in above, it may be prudent for authors to treat initial (especially non pre-registered) reports of findings as preliminary, pending a preregistered confirmation. It may even be possible to do both in the same sample. Using a crossvalidation design (Koul et al., 2018), Krämer and Rodgers (2019) randomly selected 25\% of their sample for theoretically informed (but not pre-registered) preliminary analyses, then directly confirmed (with pre-registration) these findings in the remainder of the data (the 'holdout' data). Their paper also provides examples of how to document deviations from preregistration, including sensible (but not-registered) analyses suggested during peer review. Although cross-validation requires a larger total sample, it allows researchers to both explore and confirm in a single study - allowing suicide researchers to make stronger claims with a single sample. This approach may be especially useful in clinical research (e.g., Lancaster et al., 2009), where studies are rarely strict tests of clearly specified quantitative predictions yet require confirmation before they can be relied upon for practice.

A third challenge for preregistration in suicide research is that preregistered analyses are less likely to be significant and more likely to be nuanced and complicated (e.g., Kaplan \& Irvin, 2015). For example, a researcher comparing two risk factors for suicide may find clear support only for one; pre-registration requires that the researcher discuss all planned analyses (i.e., not restricting the focus to the one 'worked'). Although this does not eliminate $p$-hacking (Claesen et al., 2019), pre-registered papers may therefore be less 'impressive'—despite being more trustworthy. Because of this, it may be desirable to combine preregistration with a registered report or results-blind peer reviewing to avoid being penalized for transparently reporting all planned analyses during peer review. Alternatively, readers and reviewers may need to be 
comfortable with less "clean" results, knowing that doing so increases the accuracy and transparency of the work.

Issue 3: Low Power. A third major source of non-replicability is low power, defined as the percentage of the time that a false null hypothesis will be rejected (i.e., a real effect will be detected; Cohen, 1988). Unfortunately, low power is a common reality in suicide research given the low base rate of suicide attempts and suicide deaths in the population ${ }^{3}$. Specifically, the population would need a suicide-mortality rate that is five times greater than the current adult suicide rate in order to achieve a positive predictive value above $10 \%$ for suicide deaths (Belsher et al., 2019).

Unfortunately, low power impairs replicability. As discussed previously, if a study has low odds of finding a true positive (low power, or an unlikely hypothesis), then any significant finding is relatively more likely to be a false positive for the simple reason that true positives are less common. This issue cannot be avoided, but it can be mitigated by reporting all resultsincluding null results - and by replicating findings, allowing false positives to be accompanied by other null results that contradict them. Issues of low power are discussed in detail by GinerSorolla et al. (2019), who dispel myths about power and offer practical guidelines for researchers, reviewers, and editors. One problem with low power is that effect size estimates have large confidence intervals; that is, a given sample result is likely to be further away from its true value in the population (Rothman \& Greenland, 2018). When combined with selective reporting and $p$-hacking, therefore, it becomes easier with low-powered studies to find and selectively report spuriously large effects. For this reason, it is recommended that researchers

\footnotetext{
${ }^{3}$ Additionally, low base rates result in distributions are often positively skewed and zero-inflated. Solutions may include oversampling for suicidality or use of statistical models that account for these distributions (GonzalezBlanks et al., 2020).
} 
discuss issues of power in their papers (now included in the APA's Journal Article Reporting Standards; Appelbaum et al., 2018) ${ }^{4}$. Because all studies have good power to detect some (large) effect size (Cohen, 1988), we suggest that researchers report the kinds of effect sizes that their studies are powered to detect (so-called power sensitivity analysis; Giner-Sorolla, 2019; see also Cohen, 1988). For the reasons discussed here, it also becomes increasingly important that researchers with small samples not $p$-hack or selectively report.

Open science solutions. Perhaps the best way to overcome low power is to avoid it, generating large datasets via collaborative, multi-site research. For example, the Military Suicide Research Consortium's (MSRC) Common Data Elements ensures that all studies funded by the MSRC includes the same set of instruments assessing suicide-related constructs such that data across studies can be later aggregated to provide the sample size to power the statistical models needed to test various research hypotheses (Plant, 2019). A repository is also in development for items used in ambulatory assessments, with the aim of testing the psychometrics of items commonly used in such studies (Kirtley et al., 2019). We highly recommend for suicide researchers using ambulatory assessments in their work to reference and contribute to this repository. Multi-site collaborations are also increasingly being used in social-personality psychology to increase the robustness of findings for these reasons (e.g., the Psychological Science Accelerator network of laboratories; https://psysciacc.org/; Moshontz et al., 2018). This trend has also spurred a 67-laboratory collaborative network in infant research, where data are similarly difficult to obtain, allowing high-powered tests of important developmental questions

\footnotetext{
${ }^{4}$ JARS also includes several tables for reporting guidelines for a variety of studies, which can be helpful to review during the design of a study (see https://apastyle.apa.org/jars/). A similar but broader resource is the EQUATOR network (https://www.equator-network.org), which serves as a hub for common reporting standards for a variety of studies such as meta-analyses, clinical trials, and more.
} 
(Bergelson et al., 2017). Although no such network yet exists for suicide research, there is no reason why existing teams could not agree to pool their efforts in this way.

Another strategy for dealing with the limited access to participants in suicide research is sequential analysis (Lakens, 2014). In sequential analysis, researchers pre-specify plans to 'peek' at the data during data collection and either stop or continue collecting data. To avoid increased false-positives, the peeks are conducted using adjusted alphas (for a tutorial, see Lakens, 2014). This enables smaller total sample sizes yet keeps one's total alpha at .05.

Issue 4: Murkiness, Missing information, and Mistakes. A lack of openness and transparency in suicide research can hinder the ability to understand and prevent suicide. In recent years, the equivocation of suicide outcomes in published studies has been brought to light (Klonsky \& May, 2014). Specifically, the definition of "suicide risk" between suicide research studies can vary greatly and may include the presence, frequency, and/or intensity of suicidal ideation; history of planned, aborted, and/or interrupted suicide attempts; suicide intent; and/or suicide deaths. In other words, the lack of clear definition between the aforementioned suicide outcomes obscures the fact that researchers are examining related yet different aspects of the suicidal experience. In a meta-analysis that established the "Suicide Constellation" of the Rorschach Inkblot Test as a well-validated indicator of suicide risk (Mihura, Meyer, Dumitrascu, \& Bombel, 2013), it was found that the authors aggregated suicide deaths, suicide attempts, and Cerebrospinal fluid levels of the serotonin metabolite 5hydroxyindoleacetic acid (5-HIAA) as if they were equivalent outcomes (Wood et al.,, 2015). As noted above, when such decisions are made after looking at results, it can be easy to inadvertently read meaning into random noise. 
Open science solutions. In order to build a strong knowledge base to understand, predict, and prevent suicide, we must be able to trust the existing literature. The sharing of research products including experimental protocols, materials used in data collection, and especially the data, codebook, and code used for data processing and analysis allows reviewers and consumers to evaluate the science at a deeper level. This is especially important with respect to methodology and analyses, as method sections in psychology often omit details deemed important by researchers (Brenninkmeijer, Derksen, \& Rietzschel, 2019). Although researchers are supposed to include enough detail to repeat an experiment (American Psychological Association, 2011; Appelbaum et al., 2018), manuscripts rarely meet this threshold. One solution is to use online supplements.

One way of sharing research products is making data openly available. Not only does this allow readers to assess the reproducibility of the results in a paper, but it also allows the checking of statistical assumptions and other criteria. Such a purpose is built into the APA Ethics Code (2017), item “8.14 Sharing Research Data for Verification”:

After research results are published, psychologists do not withhold the data on which their conclusions are based from other competent professionals who seek to verify the substantive claims through reanalysis and who intend to use such data only for that purpose, provided that the confidentiality of the participants can be protected and unless legal rights concerning proprietary data preclude their release. This does not preclude psychologists from requiring that such individuals or groups be responsible for costs associated with the provision of such information.

In clinical psychology, and particularly within suicide research, however, the sharing of particular research products may not be possible given the sensitivity of the data used in our 
work (e.g. protected health information, medical records, veteran data, PHI etc.). Fortunately, according to the Transparency and Openness Promotion guidelines (Nosek et al., 2015), data transparency can be implemented on different levels. If possible, data should be shared in a trusted repository with instructions on how to access them; if there are legal or ethical constraints (e.g., participants did not provide consent for data sharing, other legal reasons), this is also permissible so long as it is stated in the paper. There are many ethical considerations, discussed in detail elsewhere (Martone, Garcia-Castro, \& VandenBos, 2018; Meyer, 2018; Ross, Iguchi, \& Panicker, 2018). We note here, however, that participants must provide informed consent for data sharing. This can include providing permission data sharing for a period of time or indefinitely. Another issue that may arise for researchers is a reluctance to share all collected data. In this case, one common approach is to at least share the variables necessary to reproduce the results in the paper. Simmons et al. (2011) also suggest reporting that all relevant variables have been disclosed. For more discussion on ethical and privacy issues in clinical research, see Walsh et al. (2018).

If sharing the dataset used in a study is not possible due to negative constraints (e.g. funder, IRB/ethics, copyright, or other contract requirements), researchers should aim to provide as much descriptive statistics as possible for the variables used in the analyses. Furthermore, negative constraints for data sharing should be explicitly declared and justified in the manuscript (Morey et al., 2016). Finally, if the researcher is unable to share any of their data, then it would be important to provide the code, syntax, or a journal that documents the process of data collection, data processing, and data analysis that led to the reported result without the data. Data analytic reproducibility can only be achieved if the scientific community has the ability to re-run data analyses to evaluate for potential mistakes. As such, it is fundamental that researchers 
document and report each step used in data cleaning, scoring, and analysis which can be presented in the form of an RMarkdown file, SPSS Syntax, scripts, or lab journal. For example, Carpenter et al. (2019) conducted three empirical studies; their OSF (https://osf.io/jsezc/) includes datasets for all three studies, as well as analysis scripts that can be used to reproduce the results in the paper and annotated copies of the output in an HTML format (e.g., https://osf.io/3r8b7/). Although this exceeds the level of detail in the published paper, these resources are linked to, leaving nothing to the imagination of the researcher who wishes to replicate or build on the paper.

To support open and transparent research practices, the Center for Open Science developed the Open Science Framework (OSF; https://osf.io) platform which provides an open workflow that can be used across the research lifecycle such as "developing a research idea, designing a study, storing and analyzing collected data, and writing and publishing reports or papers." (Foster \& Deardorff, 2017). Furthermore, OSF serves as a platform for users to preregister their studies in addition to sharing research materials, data, unpublished results, and preprints (See Soderberg, 2018 for a guide). Finally, OSF allows researchers to create a digital object identifier (DOI) for their dataset such that they can be credited and cited when others use their data. The OSF platform has been available since 2013 but a search for the keyword "suicide" yielded only 114 projects and 47 preregistrations compared to the upwards of 7500 empirical articles published on suicide in the past 5 years.

Beyond allowing users to share their research materials and products with other users over a single platform, the community on OSF often shares blog posts, templates, as well as general support for researchers who are wanting to participate in open and transparent research practices. Furthermore, researchers can efficiently search for data that can be aggregated, form 
collaborations, and design multi-site studies on this platform. Since researchers are encouraged to stay up to date with changes in best practices for data analysis and novel techniques, access to detailed descriptions of each step taken during data analysis can also help facilitate the learning process and document why decisions were made at the time they were made - making the resulting product more useful to subsequent investigators. Having the documentation detailing each step of data processing and analysis can help reviewers and other researchers provide feedback and correct mistakes when evaluating the data analytic procedures of a study. Additionally, it is often difficult for journals to ensure that all reviewers have the statistical knowledge needed to evaluate novel data analytic approaches used in a manuscript. As such, a detailed description of each step that was taken during data analysis may assist reviewers in using their theoretical knowledge to judge the soundness of the presented results. With open access to documentation and code used for data analysis, researchers can also learn new data analytic approaches from studies that have previously implemented the approach.

\section{Replication}

Given the issues with replicability in the field, it is important for researchers to replicate existing studies that underlie focal suicide theories. Replication is fundamental to the progression of science and only findings that can be consistently reproduced under the same conditions are robust scientific discoveries (Ioannidis, 2018). As such, the direct (vs. conceptual) replication of tentative discoveries is necessary to demarcate genuine effects from spurious effects (Lilienfeld, 2018; Zwaan, Etz, Lucas, \& Donnellan, 2018). The production of novel research, that we do not intend to replicate, without testing the existing literature base also generates noise in the suicide knowledge base that may obscure our ability to recognize the genuine effects and relationships that do exist (Ioannidis, 2018). As such, we must take some time to prune away the effects and 


\section{Table 1}

\section{Recommended Open Science Practices}

\section{Minimum Practices}

1. Preregister confirmatory analyses (e.g. www.aspredicted.org)

a. If applicable, conduct confirmatory analyses for exploratory analyses using a novel dataset, a holdout sample from the original dataset, or caveat results as preliminary

2. Conduct power analysis (a priori or effect-size sensitivity); report in manuscripts

3. Transparently report analyses as exploratory or confirmatory

4. Publish preprint of the manuscript (e.g. OSF Preprints, PsyArXiv)

5. Provide access to the analysis code, research protocols, and other materials on a trusted repository (e.g. Open Science Framework)

\section{Additional Practices}

1. Provide access to the de-identified dataset on a trusted repository

2. Increase power by generating large datasets using collaborative, multi-site research

3. Engage in direct replication of existing studies in the field

relationships that cannot be replicated in order to effectively continue expanding the suicide research field. The open science practices mentioned in the previous sections of this manuscript facilitate the process of replication and ensure that the results of such replications do not end up in the file drawer. It may also be in the suicide research field's best interests to help new trainees to develop their research and analytical skills by encouraging them to evaluate and replicate extant research of interests instead of developing and testing novel research ideas. Adopting practices such as registered reports for replications can also help incentivize the practice of replication.

\section{Conclusion}

The results presented in suicide research papers are laden with clinical implications and have the ability to influence suicide risk assessment and prevention practices. As such, it is important that our studies, particularly those bearing novel and potentially impactful findings, are carefully evaluated for reproducibility. In this article, we discussed the issues underlying psychology's current replication crisis and discussed several ways that researchers can ensure 
that their work in the suicide research field are reproducible (see Table 1). This includes using open science platforms, pre-registering studies, ensuring reproducible analyses, open access to research materials and products, and conducting replication studies. We have also provided some specific recommendations for potential problems that suicide researchers may encounter as they begin to adopt open science practices. Researchers at all stages of their career can take part in the open science movement and contribute to this continuing conversation to help the suicide research field implement open science practices.

We hope that established researchers and leaders in the suicide research field will consider how they can integrate open science practices at the institutional level. This may involve modifying suicide specific journal and conference submission requirements (e.g. preregistration, open access to materials and data) to incentivize the use of open science practices. Creating a culture in which researchers must provide documentation of their data processing and analysis will reinforce transparent and open research practices. As reviewers, established researchers can start requesting access to preregistration documents, data, and code to evaluate the results presented in a manuscript before recommending it for publication.

Early career researchers can similarly help motivate change in adopting open science practices. In particular, it would be beneficial for early career researchers to consider how to implement these practices into their workflow as they establish and develop their research programs and laboratories. Engaging in open science can also allow early career researchers to establish meaningful collaborative networks in the suicide research field and deploy larger scale, multi-site, and potentially impactful work that can advance our understanding of suicide and its prevention. 
Those who are involved in the training and development of future suicide researchers can also advocate for increasing open science practices and instruction in their training programs. For example, requiring students and trainees to submit a preregistration document and code used in data processing and analysis for assignments, theses, and dissertations can help faculty members evaluate the quality and accuracy of the trainee's work. Such modifications to can also help trainees establish good open research practices and habits early in their training to help ensure that they develop into respectable suicide researchers. Finally, trainees can also contribute to the practice of open science in the suicide research field by connecting and engaging in conversations with the greater open science community through social medial platforms and requesting more training on open science practices from their advisors, programs, and student groups.

Our ability to prevent suicide deaths rests on the soundness of our science. To ensure that the suicide research field continues to progress in an efficient and meaningful manner, it is imperative that we put our houses in order. Engaging in open science will not only improve the quality and credibility of work in the field of suicide research but it will also help optimize our ability to predict and prevent suicide deaths. By simplifying and outlining the steps needed to engage in open research and providing guidelines on how to apply such steps specifically in the field of suicide, we hope to motivate and encourage more suicide researchers to embrace open and transparent research practices in their work. This will provide us with the solid foundation that is needed to advance us towards our common goal of preventing deaths by suicide. 


\section{References}

American Psychological Association. (2011). Publication manual of the American Psychological Association (6th ed). Washington, DC: American Psychological Association.

American Psychological Association. (2017). Ethical principles of psychologists and code of conduct. Retrieved from https://www.apa.org/ethics/code/

Appelbaum, M., Cooper, H., Kline, R. B., Mayo-Wilson, E., Nezu, A. M., \& Rao, S. M. (2018). Journal article reporting standards for quantitative research in psychology: The APA publications and communications board task force report. American Psychologist, 73, 325. http://dx.doi.org/10.1037/amp0000191

Back, M., Hendriks, F., Schönbrodt, F., \& the Network for Open Science Initiatives. (2017). Open science made easy: 7 steps towards transparent and reproducible research [Brochure]. Retrieved from https://osf.io/hktmf/

Badenes-Ribera, L., Frias-Navarro, D., Iotti, B., Bonilla-Campos, A., \& Longobardi, C. (2016). Misconceptions of the p-value among Chilean and Italian Academic Psychologists. Frontiers in Psychology, 7. https://doi.org/10.3389/fpsyg.2016.01247

Belsher, B. E., Smolenski, D. J., Pruitt, L. D., Bush, N. E., Beech, E. H., Workman, D. E., ... Skopp, N. A. (2019). Prediction models for suicide attempts and deaths: A systematic review and simulation. JAMA Psychiatry, 76(6), 642-651. https://doi.org/10.1001/jamapsychiatry.2019.0174

Bem, D. (1987). Writing the empirical journal article. In J. M. Darley \& M. P. Zanna (Eds.), The compleat academic: A career guide (1st ed, pp. 171-201). Mahwah, NJ: Lawrence Erlbaum. 
Benjamini, Y., \& Hochberg, Y. (1995). Controlling the false discovery rate: A practical and powerful approach to multiple testing. Journal of the Royal Statistical Society, 57(1), 289-300.

Bergelson, E., Bergmann, C., Byers-Heinlein, K., Cristia, A., Cusack, R., Dyck, K., ... Yurovsky, D. (2017). Quantifying sources of variability in infancy research using the infant-directed speech preference [Preprint]. https://doi.org/10.31234/osf.io/s98ab

Brenninkmeijer, J., Derksen, M., \& Rietzschel, E. (2019). Informal laboratory practices in psychology. Collabra: Psychology, 5(1), 45. https://doi.org/10.1525/collabra.221

Carpenter, T. P., Pogacar, R., Pullig, C., Kouril, M., Aguilar, S., LaBouff, J., ... Chakroff, A. (2019). Survey-software implicit association tests: A methodological and empirical analysis. Behavior Research Methods. Advance online publication. https://doi.org/10.3758/s13428-019-01293-3

Carter, E. C., Schönbrodt, F. D., Gervais, W. M., \& Hilgard, J. (2019). Correcting for bias in psychology: A comparison of meta-analytic methods. Advances in Methods and Practices in Psychological Science, 2(2), 115-144. https://doi.org/10.1177/2515245919847196

Chambers, C. D., Feredoes, E., Muthukumaraswamy, S. D., \& Etchells, P. (2014). Instead of "playing the game" it is time to change the rules: Registered reports at AIMS Neuroscience and beyond. AIMS Neuroscience, 1(1), 4-17. doi: 10.3934/Neuroscience2014.1. 4

Claesen, A., Gomes, S. L. B. T., Tuerlinckx, F., \& Vanpaemel, W. (2019). Preregistration: Comparing dream to reality [Preprint]. https://doi.org/10.31234/osf.io/d8wex 
Cohen, J. (1988). Statistical power analysis for the behavioral sciences (2nd ed.). Hillsdale, NJ: Erlbaum.

Coyne, J. C. (2016). Replication initiatives will not salvage the trustworthiness of psychology. BMC Psychology, 4(1), 1-11. https://doi.org/10.1186/s40359-016-0134-3

Crüwell, S., van Doorn, J., Etz, A., Makel, M. C., Moshontz, H., Niebaum, J. C., ... SchulteMecklenbeck, M. (2018, November 16). 7 easy steps to open science: An annotated reading list [Preprint]. https://doi.org/10.31234/osf.io/cfzyx

DeHaven, A. (2017, May 23). Preregistration: A plan, not a prison. Retrieved from: https://cos.io/blog/preregistration-plan-not-prison/

Dang, J., Zerhouni, O., Imhoff, R., Jia, L., Giacomantonio, M., Sevincer, A. T., ... Schiöth, H. B. (2019). Multi-lab replication reveals a small but significant ego depletion effect [Preprint]. https://doi.org/10.31234/osf.io/cjgru

Franklin, J. C., Ribeiro, J. D., Fox, K. R., Bentley, K. H., Kleiman, E. M., Huang, X., ... Nock, M. K. (2017). Risk factors for suicidal thoughts and behaviors: A meta-analysis of 50 years of research. Psychological Bulletin, 143(2), 187-232. https://doi.org/10.1037/bul0000084

Foster, E. D., \& Deardorff, A. (2017). Open Science Framework (OSF). Journal of the Medical Library Association, 105(2), 203-206. https://doi.org/10.5195/jmla.2017.88

Giner-Sorolla, R., Schoemann, A. M., Montoya, A. K., Conrique, B. G., Carpenter, T. P., Reifman, A., ... Bostyn, D. H. (2019). Power to detect what? Considerations for planning and evaluating sample size [Preprint]. https://osf.io/jnmya/

Grand, J. A., Rogelberg, S. G., Banks, G. C., Landis, R. S., \& Tonidandel, S. (2018). From outcome to process focus: Fostering a more robust psychological science through 
registered reports and results-blind reviewing. Perspectives on Psychological Science, 13(4), 448-456. https://doi.org/10.1177/1745691618767883

Greenland, S., Senn, S. J., Rothman, K. J., Carlin, J. B., Poole, C., Goodman, S. N., \& Altman, D. G. (2016). Statistical tests, $p$-values, confidence intervals, and power: A guide to misinterpretations. European Journal of Epidemiology, 31(4), 337-350. https://doi.org/10.1007/s10654-016-0149-3

O’Donnell, M., Nelson, L. D., Ackermann, E., Aczel, B., Akhtar, A., Aldrovandi, S., ... Zrubka, M. (2018). Registered replication report: Dijksterhuis and van Knippenberg (1998). Perspectives on Psychological Science, 13(2), 268-294. https://doi.org/10.1177/1745691618755704

Easterbrook, P. J., Gopalan, R., Berlin, J. A., \& Matthews, D. R. (1991). Publication bias in clinical research. The Lancet, 337(8746), 867-872. https://doi.org/10.1016/01406736(91)90201-Y

Ebersole, C. R., Atherton, O. E., Belanger, A. L., Skulborstad, H. M., Allen, J. M., Banks, J. B., ... Nosek, B. A. (2016). Many Labs 3: Evaluating participant pool quality across the academic semester via replication. Journal of Experimental Social Psychology, 67, 6882. https://doi.org/10.1016/j.jesp.2015.10.012

Forscher, P. S., Taylor, V. J., Cavagnaro, D., Lewis, N. A., Moshontz, H., Batres, C., ... Chartier, C. R. (2019). A multi-site examination of stereotype threat in black college students across varying operationalizations [Preprint]. https://doi.org/10.31234/osf.io/6hju9

Hagger, M. S., Chatzisarantis, N. L. D., Alberts, H., Anggono, C. O., Batailler, C., Birt, A. R., ... Zwienenberg, M. (2016). A multilab preregistered replication of the ego-depletion effect. 
Perspectives on Psychological Science, 11(4), 546-573. https://doi.org/10.1177/1745691616652873

Head, M. L., Holman, L., Lanfear, R., Kahn, A. T., \& Jennions, M. D. (2015). The extent and consequences of $p$-hacking in science. PLoS Biol, 13(3), e1002106. https://doi.org/10.1371/journal.pbio.1002106

Ioannidis, J. P. A. (2005). Why most published research findings are false. PLoS Med, 2(8), e124. https://doi.org/10.1371/journal.pmed.0020124

Ioannidis, J. P. A. (2018). Why replication has more scientific value than original discovery. Behavioral and Brain Sciences, 41, e137. https://doi.org/10.1017/S0140525X18000729

Kaplan, R. M., \& Irvin, V. L. (2015). Likelihood of null effects of large NHLBI clinical trials has increased over time. PLOS ONE, 10(8), e0132382. https://doi.org/10.1371/journal.pone.0132382

Kerr, N. L. (1998). HARKing: Hypothesizing after the results are known. Personality and Social Psychology Review, 2, 196-217. https://doi.org/10.1207/s15327957pspr0203_4

Kirk, R. E. (2008). Statistics: An introduction (5th ed.). Belmont, CA: Thomson Wadsworth.

Kirtley, O. J., Lafit, G., Achterhof, R., Hiekkaranta, A. P., \& Myin-Germeys, I. (2019, April 10). Making the black box transparent: A template and tutorial for (pre-)registration of studies using Experience Sampling Methods (ESM). https://doi.org/10.31234/osf.io/seyq7

Kirtley, O.J., Hiekkaranta, A.P., Kunkels, Y.K., Verhoeven, D., Nierop, M.V., \& MyinGermeys, I. (2018, December 4). The Experience Sampling Method (ESM) Item Repository. https://doi.org/10.17605/OSF.IO/KG376 
Klein, O., Hardwicke, T. E., Aust, F., Breuer, J., Danielsson, H., Hofelich Mohr, A., ... Frank, M. C. (2018). A practical guide for transparency in psychological science. Collabra: Psychology, 4(1), 20. DOI: http://doi.org/10.1525/collabra.158

Klonsky, E. D., \& May, A. M. (2014). Differentiating suicide attempters from suicide ideators: A critical frontier for suicidology research. Suicide and Life-Threatening Behavior. https://doi.org/10.1111/sltb.12068

Krämer, M. D., \& Rodgers, J. L. (2019). The impact of having children on domain-specific life satisfaction: A quasi-experimental longitudinal investigation using the Socio-Economic Panel (SOEP) data. Journal of Personality and Social Psychology. [Advance online publication]. https://doi.org/10.1037/pspp0000279

Lakens, D. (2014). Performing high-powered studies efficiently with sequential analyses. European Journal of Social Psychology, 44(7), 701-710. https://doi.org/10.1002/ejsp.2023

Lakens, D. (2017). Equivalence tests: A practical primer for $t$-tests, correlations, and metaanalyses. Social Psychological and Personality Science, 8 (4), 355-362. https://doi.org/10.1177/1948550617697177

Lancaster, S. L., Melka, S. E., \& Rodriguez, B. F. (2009). A factor analytic comparison of five models of PTSD symptoms. Journal of Anxiety Disorders, 23(2), 269-274. https://doi.org/10.1016/j.janxdis.2008.08.001

LeBel, E. P., Berger, D., Campbell, L., \& Loving, T. J. (2017). Falsifiability is not optional. Journal of Personality and Social Psychology, 113(2), 254-261. https://doi.org/10.1037/pspi0000106 
Lilienfeld, S. O. (2018). Direct replication and clinical psychological science. Behavioral and Brain Sciences, 41, e140. https://doi.org/10.1017/S0140525X18000754

Martone, M. E., Garcia-Castro, A., \& VandenBos, G. R. (2018). Data sharing in psychology. American Psychologist, 73(2), 111-125. https://doi.org/10.1037/amp0000242

Masson, M. E. J. (2011). A tutorial on a practical Bayesian alternative to null-hypothesis significance testing. Behavior Research Methods, 43(3), 679-690. https://doi.org/10.3758/s13428-010-0049-5

Merton, R. K. (1942). A note on science and democracy. Journal of Legal and Political Sociology, 1, 115-128.

Mertens, G., \& Krypotos, A.-M. (2019). Preregistration of analyses of preexisting data [Preprint]. https://doi.org/10.31234/osf.io/ph4q7

Meyer, M. N. (2018). Practical Tips for Ethical Data Sharing. Advances in Methods and Practices in Psychological Science, 1(1), 131-144. https://doi.org/10.1177/2515245917747656

Mihura, J. L., Meyer, G. J., Dumitrascu, N. \& Bombel, G. (2013). The validity of individual Rorschach variables: Systematic reviews and meta-analyses of the comprehensive system. Psychological Bulletin, 139(3), 548-605. doi: 0.1037/a0029406

Moshontz, H., Campbell, L., Ebersole, C. R., IJzerman, H., Urry, H. L., Forscher, P. S., ... Chartier, C. R. (2018). The psychological science accelerator: Advancing psychology through a distributed collaborative network. Advances in Methods and Practices in Psychological Science, 1(4), 501-515. https://doi.org/10.1177/2515245918797607

Morey, R. D., Chambers, C. D., Etchells, P. J., Harris, C. R., Hoekstra, R., Lakens, D., Lewandowsky, S., Morey, C. C., Newman, D. P., Schönbrodt, F., Vanpaemel, W., 
Wagenmakers, E.-J., \& Zwaan, R. A. (2016). The peer reviewers' openness initiative: Incentivising open research practices through peer review. Royal Society Open Science, 3, 1-7. DOI: https://doi.org/10.1098/rsos.150547

National Institutes of Health (2016). NIH Policy on the Dissemination of NIH-Funded Clinical Trial Information. https://grants.nih.gov/grants/guide/notice-files/NOT-OD-16-149.html

Neyman, J., \& Pearson, E. S. (1933). The testing of statistical hypotheses in relation to probabilities a priori. Mathematical Proceedings of the Cambridge Philosophical Society, 29(4), 492-510. https://doi.org/10.1017/S030500410001152X

Nock, M. K., Park, J. M., Finn, C. T., Deliberto, T. L., Dour, H. J., \& Banaji, M. R. (2010). Measuring the suicidal mind: Implicit cognition predicts suicidal behavior. Psychological Science, 21, 511-517. https://doi.org/10.1177/0956797610364762

Nosek, B. A., Alter, G., Banks, G. C., Borsboom, D., Bowman, S. D., Breckler, S. J., ... Yarkoni, T. (2015). Promoting an open research culture. Science, 348(6242), 1422-1425. doi: $10.1126 /$ science.aab2374

Nosek, B. A., \& Bar-Anan, Y. (2012). Scientific utopia: I. Opening scientific communication. Psychological Inquiry, 23(3), 217-243. https://doi.org/10.1080/1047840X.2012.692215

Nosek, B. A., Spies, J. R., \& Motyl, M. (2012). Scientific utopia: II. Restructuring incentives and practices to promote truth over publishability. Perspectives on Psychological Science, 7(6), 615-631. https://doi.org/10.1177/1745691612459058

Open Science Collaboration. (2015). Estimating the reproducibility of psychological science. Science, 349(6251), aac4716. https://doi.org/10.1126/science.aac4716 
Pirkis, J. (2020). Strengthening the evidence base in suicide prevention: Systematic reviews and registered reports [Editorial]. Crisis: The Journal of Crisis Intervention and Suicide Prevention, 41(2), 77-81. https://doi.org/10.1027/0227-5910/a000693

Plant, A. (2019, June 28). MSRC Common Data Elements (CDE). Retrieved from: https://msrc.fsu.edu/news/msrc-common-data-elements-cde

Popper, K. R. (1968). Conjectures and refutations: The growth of scientific knowledge. New York, NY: Harper \& Row

Pu, X., Zhu, L., Kay, M., \& Conrad, F. (2019). Designing for preregistration in practice: Multiple norms and purposes [Preprint]. https://doi.org/10.31219/osf.io/w5ked

Rothman, K. J., \& Greenland, S. (2018). Planning study size based on precision rather than power. Epidemiology, 29(5), 599-603. https://doi.org/10.1097/EDE.0000000000000876

Ross, M. W., Iguchi, M. Y., \& Panicker, S. (2018). Ethical aspects of data sharing and research participant protections. American Psychologist, 73(2), 138-145. https://doi.org/10.1037/amp0000240

Silberzahn, R., Uhlmann, E. L., Martin, D. P., Anselmi, P., Aust, F., Awtrey, E., ... Nosek, B. A. (2018). Many analysts, one data set: Making transparent how variations in analytic choices affect results. Advances in Methods and Practices in Psychological Science, 1(3), 337-356. https://doi.org/10.1177/2515245917747646

Simmons, J. P., Nelson, L. D., \& Simonsohn, U. (2011). False-positive psychology: Undisclosed flexibility in data collection and analysis allows presenting anything as significant. Psychological Science, 22, 1359-1366. https://doi.org/10.1177/0956797611417632 
Soderberg, C. K. (2018). Using OSF to share data: A step-by-step guide. Advances in Methods and Practices in Psychological Science, 1(1), 115-120.

https://doi.org/10.1177/2515245918757689

Szucs, D., \& Ioannidis, J. P. A. (2017). Empirical assessment of published effect sizes and power in the recent cognitive neuroscience and psychology literature. PLOS Biology, 15, e2000797. https://doi.org/10.1371/journal.pbio.2000797

Tackett, J. L., Lilienfeld, S. O., Patrick, C. J., Johnson, S. L., Krueger, R. F., Miller, J. D., ... Shrout, P. E. (2017). It's time to broaden the replicability conversation: Thoughts for and from clinical psychological science. Perspectives on Psychological Science, 12(5), 742-756. https://doi.org/10.1177/1745691617690042

Tello, N., Harika-Germaneau, G., Serra, W., Jaafari, N., \& Chatard, A. (2020). Forecasting a fatal decision: Direct replication of the predictive validity of the Suicide-Implicit Association Test. Psychological Science, 31(1), 65-74.

Uhlmann, E. L., Ebersole, C. R., Chartier, C. R., Errington, T. M., Kidwell, M. C., Lai, C. K., ... Nosek, B. A. (2019). Scientific utopia III: Crowdsourcing science. Perspectives on Psychological Science, 14(5), 711-733. https://doi.org/10.1177/1745691619850561

van 't Veer, A. E., \& Giner-Sorolla, R. (2016). Preregistration in social psychology-A discussion and suggested template. Journal of Experimental Social Psychology, 67, 2-12. https://doi.org/10.1016/j.jesp.2016.03.004

Walsh, C. G., Xia, W., Li, M., Denny, J. C., Harris, P. A., \& Malin, B. A. (2018). Enabling openscience initiatives in clinical psychology and psychiatry without sacrificing patients' privacy: Current practices and future challenges. Advances in Methods and Practices in Psychological Science, 1(1), 104-114. https://doi.org/10.1177/2515245917749652 
Wagenmakers, E.-J., Beek, T., Dijkhoff, L., Gronau, Q. F., Acosta, A., Adams, R. B., ... Zwaan, R. A. (2016). Registered replication report: Strack, Martin, \& Stepper (1988). Perspectives on Psychological Science, 11(6), 917-928. https://doi.org/10.1177/1745691616674458

Werner, K. M., Milyavskaya, M., Klimo, R., \& Levine, S. L. (2019). Examining the unique and combined effects of grit, trait self-control, and conscientiousness in predicting motivation for academic goals: A commonality analysis. Journal of Research in Personality, 81, 168-175. https://doi.org/10.1016/j.jrp.2019.06.003

Weston, S. J., Ritchie, S. J., Rohrer, J. M., \& Przybylski, A. K. (2018). Recommendations for increasing the transparency of analysis of pre-existing datasets [Preprint]. https://doi.org/10.31234/osf.io/zmt3q

Wood, J. M., Garb, H. N., Nezworski, M. T., Lilienfeld, S. O. \& Duke, M. C. (2015) A second look at the validity of widely used Rorschach indices: Comment on Mihura, Meyer, Dumitrascu, and Bombel (2013). Psychological Bulletin, 141(1), 236-249. http://dx.doi.org/10.1037/a0036005

Zwaan, R. A., Etz, A., Lucas, R. E., \& Donnellan, M. B. (2017). Making replication mainstream. Behavioral and Brain Sciences, 41, e120. https://doi.org/10.1017/S0140525X17001972 